\title{
Statistical Order Convergence and Statistically Relatively Uniform Convergence in Riesz Spaces
}

\author{
Xuemei Xue $\mathbb{D}^{1,2}$ and Jian Tao $\mathbb{D i D}^{1}$ \\ ${ }^{1}$ School of Mathematics and Statistics, Northeast Normal University, Changchun, Jilin 130024, China \\ ${ }^{2}$ School of Mathematical Sciences, Xiamen University, Xiamen 361005, China \\ Correspondence should be addressed to Jian Tao; taoj@nenu.edu.cn
}

Received 25 January 2018; Revised 25 February 2018; Accepted 6 March 2018; Published 15 April 2018

Academic Editor: Yoshihiro Sawano

Copyright (C) 2018 Xuemei Xue and Jian Tao. This is an open access article distributed under the Creative Commons Attribution License, which permits unrestricted use, distribution, and reproduction in any medium, provided the original work is properly cited.

A new concept of statistically $e$-uniform Cauchy sequences is introduced to study statistical order convergence, statistically relatively uniform convergence, and norm statistical convergence in Riesz spaces. We prove that, for statistically e-uniform Cauchy sequences, these three kinds of convergence for sequences coincide. Moreover, we show that the statistical order convergence and the statistically relatively uniform convergence need not be equivalent. Finally, we prove that, for monotone sequences in Banach lattices, the norm statistical convergence coincides with the weak statistical convergence.

\section{Introduction}

The first idea of statistical convergence goes back to Zygmund's monograph [1], where Zygmund called it almost convergence. The concept of statistical convergence was formally introduced by Steinhaus [2] and Fast [3] and later reintroduced by Schoenberg [4]. Statistical convergence, as a generalization of the usual notion of convergence, has attracted much attention since it was introduced over sixty years ago. This concept has been applied in various areas such as number theory [5], trigonometric series [1], summability theory [6], measure theory [7], and probability theory [8]. Furthermore, generalizations have appeared in Hausdorff topological spaces and function spaces [9], locally convex spaces [10,11], and Banach spaces [12].

The concepts of order convergence and relatively uniform convergence are two fundamental concepts and basic tools in the study of Riesz spaces. The two big books $[13,14]$ present a good and detailed investigation of these two concepts. Sençimen and Pehlivan [15] introduced the concept of statistical order convergence, which is a natural generalization of order convergence. Meanwhile, some basic definitions and results are established in [15]. Ercan [16] introduced the notion of statistically $u$-uniformly convergent sequences in
Riesz spaces, which is a statistical analogue of $u$-uniformly convergent sequences.

The aim of our present paper is to continue to develop the theory of statistical order convergence and statistically $u$-uniform convergence. The present paper is organized as follows.

In Section 2, we first establish a useful characterization of statistically $e$-uniform convergence, which is a statistical analogue of an equivalent definition of $e$-uniform convergence. Secondly, we prove that a statistically $e$-uniformly convergent sequence almost coincides with an $e$-uniformly convergent sequence. As a corollary, we show that every monotone statistically relatively uniformly convergent sequence is relatively uniformly convergent. In [16], Ercan introduced the notion of statistically $e$-uniform pre-Cauchy sequence and proved that each statistically $e$-uniform pre-Cauchy sequence admits an $e$-uniform Cauchy subsequence. In this section, we introduce the notion of statistically $e$-uniform Cauchy sequence and prove that a sequence $\left(x_{n}\right)_{n \in \mathbb{N}}$ is statistically $e$-uniform Cauchy if and only if there exists a subset $K=\left\{k_{j}: j \in \mathbb{N}\right\}$ of $\mathbb{N}$ with $\delta(K)=1$ such that $\left(x_{k_{j}}\right)_{j \in \mathbb{N}}$ is an $e$-uniform Cauchy sequence. Using this result, we obtain that every monotone statistically $e$-uniform Cauchy sequence is $e$-uniform Cauchy. Furthermore, we show that every statistically $e$-uniform 
Cauchy sequence is statistically $e$-uniformly pre-Cauchy. Finally, we prove that every monotone statistically $e$-uniform Cauchy sequence in a Dedekind $\sigma$-complete Riesz space $E$ is statistically $e$-uniformly convergent.

Section 3 is concerned with close relationship among statistically relatively uniform convergence, statistical order convergence, and norm statistical convergence. In this section, we prove that, for uniform Cauchy sequences, the relatively uniform convergence coincides with the statistical order convergence. Similarly, we also show that the statistically relatively uniform convergence coincides with the statistical order convergence for statistically uniform Cauchy sequences. Moreover, we show that the statistical order convergence and the statistically relatively uniform convergence need not be equivalent. The rest of this section is devoted to norm statistical convergence. We prove that, for statistically uniform Cauchy sequences, the statistically relatively uniform convergence also coincides with the norm statistical convergence. Finally, we show that the norm statistical convergence and the weakly statistical convergence are equivalent for monotone sequences.

Notation 1. Throughout the paper, $\mathbb{N}$ will denote the set of all positive integers and $\mathbb{R}$ will denote the set of all real numbers. Let $E$ be a Riesz space. The set of all positive elements of $E$ is denoted by $E_{+}$. If $K$ is a subset of $\mathbb{N}$, then $K_{n}$ denotes the set $\{k \in K: k \leq n\}$ and $\left|K_{n}\right|$ denotes the number of elements in $K_{n}$. The natural density of $K$ is defined by $\delta(K)=\lim _{n \rightarrow \infty}\left(\left|K_{n}\right| / n\right)$. Let $\left(x_{n}\right)_{n \in \mathbb{N}}$ be a sequence in a Riesz space $E$. If $\left(x_{n}\right)_{n \in \mathbb{N}}$ is increasing (decreasing), we shall write $x_{n} \uparrow\left(x_{n} \downarrow\right)$. If, moreover, $\sup _{n \in \mathbb{N}} x_{n}=x\left(\inf _{n \in \mathbb{N}} x_{n}=x\right)$ exists, then we denote this by $x_{n} \uparrow x\left(x_{n} \downarrow x\right)$. Given a sequence $\left(x_{n}\right)_{n \in \mathbb{N}}$ in a normed Riesz space $E$ and an infinite subset $A \subseteq \mathbb{N}$, we say that $\left(x_{n}\right)_{n \in \mathbb{N}}$ converges in norm to $x \in E$ along $A$ if, for every $\epsilon>0$, there exists a natural number $N$ such that for each $n>N, n \in A$ we have $\left\|x_{n}-x\right\|<\epsilon$. We denote this by $\lim _{n \in A} x_{n}=x$. If a sequence $\left(x_{n}\right)_{n \in \mathbb{N}}$ in a Riesz space $E$ satisfies property $P$ for all $n$ except a natural density zero set, then we say that $\left(x_{n}\right)_{n \in \mathbb{N}}$ satisfies property $P$ for almost all $n$ and we abbreviate this by a.a.n. [17]. A Riesz space $E$ is called Dedekind $\sigma$-complete if every nonempty finite or countable subset which is order bounded from above has a supremum and every nonempty finite or countable subset which is order bounded from below has an infimum.

The reader is referred to $[13,14]$ for more details about Riesz spaces.

\section{Statistically Relatively Uniform Convergence}

Let $E$ be a Riesz space and let $e \in E_{+}$. A sequence $\left(x_{n}\right)_{n \in \mathbb{N}}$ in $E$ is said to converge $e$-uniformly to $x \in E[13$, Definition 39.1] whenever, for every $\epsilon>0$, there exists a natural number $N$ such that $\left|x_{n}-x\right| \leq \epsilon e$ holds for all $n \geq N$. We denote this by $x_{n} \stackrel{\mathrm{ru}}{\longrightarrow} x(e)$. It is said that the sequence $\left(x_{n}\right)_{n \in \mathbb{N}}$ in $E$ converges relatively uniformly to $x$ whenever $\left(x_{n}\right)_{n \in \mathbb{N}}$ converges $e$-uniformly to $x$ for some $e \in E_{+}$. We shall write $x_{n} \rightarrow x(\mathrm{ru})$.
It should be noted that, in an Archimedean space $E$, a sequence $x_{n} \stackrel{\text { ru }}{\longrightarrow} x(e)$ if and only if there exists a sequence $\left(\epsilon_{n}\right)_{n \in \mathbb{N}}$ of positive reals with $\epsilon_{n} \downarrow 0$ such that $\left|x_{n}-x\right| \leq \epsilon_{n} e$ for all $n \in \mathbb{N}$. See [18, Definition 2.23, p. 24] for this equivalent definition.

Ercan [16] modified the definition of $e$-uniformly convergent sequences in Riesz spaces as follows.

Definition 2 (see [16]). Let $E$ be a Riesz space and $e \in E_{+}$. A sequence $\left(x_{n}\right)_{n \in \mathbb{N}}$ in $E$ is said to be statistically $e$-uniformly convergent to $x \in E$ if

$$
\lim _{n \rightarrow \infty} \frac{1}{n}\left|\left\{k: k \leq n,\left(\left|x_{k}-x\right|-\epsilon e\right)^{+}>0\right\}\right|=0
$$

for each $\epsilon>0$. Equivalently, $\left|x_{n}-x\right| \leq \epsilon e$, a.a.n. for each $\epsilon>0$. We write $x_{n} \stackrel{\text { st }}{\rightarrow} x(e)$. We say that a sequence $\left(x_{n}\right)_{n \in \mathbb{N}}$ in $E$ converges statistically relatively uniformly to $x$ provided that $x_{n} \stackrel{\text { st }}{\rightarrow} x(e)$ for some $e \in E_{+}$. We write $x_{n} \stackrel{\text { sru }}{\longrightarrow} x$.

The following well-known lemma about sets of natural density is the key to establishing many results regarding statistically convergent sequences.

Lemma 3 (see [6]). Let $\left\{A_{i}\right\}_{i \in I}$ be a countable collection of subsets of $\mathbb{N}$ such that $\delta\left(A_{i}\right)=1$ for each $i \in I$. Then there is a subset $A \subseteq \mathbb{N}$ such that $\delta(A)=1$ and $\left|A \backslash A_{i}\right|<\infty$ for all $i \in I$.

The following characterization of statistically $e$-uniform convergence is useful and will be used in the sequel.

Theorem 4. Let $E$ be an Archimedean Riesz space and $\in \in E_{+}$. The following are equivalent for a sequence $\left(x_{n}\right)_{n \in \mathbb{N}}$ in $E$ and $x \in E$ :

(1) $x_{n} \stackrel{s t}{\rightarrow} x(e)$.

(2) There exists a sequence $\left(\epsilon_{n}\right)_{n \in \mathbb{N}}$ of positive reals with $\epsilon_{n} \downarrow 0$ such that

$$
\left|x_{n}-x\right| \leq \epsilon_{n} e, \quad \text { a.a.n. }
$$

Proof.

$(1) \Rightarrow(2)$. Suppose that $x_{n} \stackrel{\text { st }}{\rightarrow} x(e)$. For each $k \in \mathbb{N}$, we set

$$
A_{k}=\left\{n \in \mathbb{N}:\left|x_{n}-x\right| \leq \frac{1}{k} e\right\} \text {. }
$$

Then $\delta\left(A_{k}\right)=1$ and $A_{k} \supseteq A_{k+1}$ for each $k \in \mathbb{N}$.

Let $E_{e}$ be the principal ideal generated by the singleton $\{e\}$. We define

$$
\|x\|_{e}=\inf \{\lambda>0:|x| \leq \lambda e\}
$$

for each $x \in E_{e}$. Then $\|\cdot\|_{e}$ is a lattice norm on $E_{e}$ and $|x| \leq$ $\|x\|_{e} e$ for all $x \in E_{e}$.

By Lemma 3, we get a subset $A \subseteq \mathbb{N}$ such that $\delta(A)=1$ and $\left|A \backslash A_{k}\right|<\infty$ for all $k \in \mathbb{N}$.

Claim $4 . \lim _{n \in A \cap A_{1}}\left\|x_{n}-x\right\|_{e}=0$. 
Indeed, for every $\epsilon>0$, we choose $k_{0} \in \mathbb{N}$ with $1 / k_{0}<\epsilon$. We set $N=\max _{n \in A \backslash A_{k_{0}}} n$. Then, for each $n>N, n \in A$, we have

$$
\left\|x_{n}-x\right\|_{e} \leq \frac{1}{k_{0}}<\epsilon .
$$

Let $A \cap A_{1}=\left\{k_{j}: j \in \mathbb{N}\right\}$. Then $\delta\left(A \cap A_{1}\right)=1$. We let $\epsilon_{n}=\sup _{j \geq n}\left\|x_{k_{j}}-x\right\|_{e}$ for each $n \in \mathbb{N}$. Then $\epsilon_{n} \downarrow 0$ and

$$
\left|x_{k_{n}}-x\right| \leq\left\|x_{k_{n}}-x\right\|_{e} e \leq \epsilon_{n} e, \quad n=1,2, \ldots
$$

Let $\eta_{n}=\epsilon_{j}$ for $k_{j-1}<n \leq k_{j}(j=1,2, \ldots)$, where $k_{0}=0$. Then $\eta_{n} \downarrow 0$ and

$$
\left|x_{k_{j}}-x\right| \leq \epsilon_{j} e=\eta_{k_{j}} e, \quad j=1,2, \ldots
$$

This implies

$$
A \cap A_{1} \subseteq\left\{n \in \mathbb{N}:\left|x_{n}-x\right| \leq \eta_{n} e\right\}
$$

Hence

$$
\delta\left(\left\{n \in \mathbb{N}:\left|x_{n}-x\right| \leq \epsilon_{n} e\right\}\right)=1 .
$$

(2) $\Rightarrow(1)$. Let $\left(\epsilon_{n}\right)_{n \in \mathbb{N}}$ be the sequence of positive reals as stated in (2). Let $K=\left\{n \in \mathbb{N}:\left|x_{n}-x\right| \leq \epsilon_{n} e\right\}$ and $\epsilon>0$. Choose $k_{0}$ with $\epsilon_{k_{0}}<\epsilon$. Then, for $n \in K$ and $n>k_{0}$, we have

$$
\left|x_{n}-x\right| \leq \epsilon_{n} e \leq \epsilon_{k_{0}} e \leq \epsilon e .
$$

Since $\delta\left(\left\{n \in K: n>k_{0}\right\}\right)=1$, we get

$$
\delta\left(\left\{n \in \mathbb{N}:\left|x_{n}-x\right| \leq \epsilon e\right\}\right)=1
$$

This completes the proof.

Note that a subsequence of a statistically relatively uniform convergent sequence need not be statistically relatively uniform convergent and a statistically relatively uniform convergent sequence need not be relatively uniform convergent. These facts can be seen in the following easy example.

Example 5. Let $\left(x_{n}\right)_{n \in \mathbb{N}}$ be a sequence in $\mathbb{R}$ defined by

$$
x_{n}= \begin{cases}n, & n=k^{2} \quad(k \in \mathbb{N}) \\ \frac{1}{n}, & \text { otherwise. }\end{cases}
$$

Then, $x_{n}=1 / n$, a.a.n. and hence, by Theorem 4 , we have $x_{n} \stackrel{\text { st }}{\rightarrow} 0$ (1). But, it is obvious that the subsequence $\left(x_{n}\right)_{n \in K}$ of $\left(x_{n}\right)_{n \in \mathbb{N}}$, where $K=\left\{k^{2}: k \in \mathbb{N}\right\}$, is not statistically relatively uniform convergent. Since the sequence $\left(x_{n}\right)_{n \in \mathbb{N}}$ is not order bounded, $\left(x_{n}\right)_{n \in \mathbb{N}}$ is not relatively uniform convergent.

Remark 6. Statistically relatively uniform convergence is stable; that is, if $x_{n} \stackrel{\text { sru }}{\longrightarrow} 0$, then there exists a sequence $\left(\lambda_{n}\right)_{n \in \mathbb{N}}$ of reals with $0 \leq \lambda_{n} \uparrow \infty$ such that $\lambda_{n} x_{n} \stackrel{\text { sru }}{\longrightarrow} 0$. Indeed, there exist $e \in E_{+}$and a sequence $\left(\epsilon_{n}\right)_{n \in \mathbb{N}}$ of reals with $\epsilon_{n} \downarrow 0$ such that

$$
\left|x_{n}\right| \leq \epsilon_{n} e, \quad \text { a.a.n. }
$$

That is,

$$
\frac{1}{\sqrt{\epsilon_{n}}}\left|x_{n}\right| \leq \sqrt{\epsilon_{n}} e, \quad \text { a.a.n. }
$$

Hence,

$$
\frac{1}{\sqrt{\epsilon_{n}}} x_{n} \stackrel{\text { sru }}{\longrightarrow} 0 \text {. }
$$

We give another useful characterization of statistically $e$ uniform convergence.

Theorem 7. Let $E$ be an Archimedean Riesz space and $e \in E_{+}$. The following are equivalent for a sequence $\left(x_{n}\right)_{n \in \mathbb{N}}$ in $E$ and $x \in E$ :

(1) $x_{n} \stackrel{s t}{\rightarrow} x(e)$.

(2) There exists a subset $K=\left\{k_{j}: j \in \mathbb{N}\right\}$ of $\mathbb{N}$ with $\delta(K)=$ 1 such that $x_{k_{j}} \stackrel{r u}{\longrightarrow} x(e)$.

(3) There exists a sequence $\left(y_{n}\right)_{n \in \mathbb{N}}$ in $E$ such that $x_{n}=$ $y_{n}$ a.a.n. and $y_{n} \stackrel{r u}{\longrightarrow} x(e)$.

Proof. The implication (1) $\Rightarrow$ (2) follows from Theorem 4 .

(2) $\Rightarrow(3)$. Assume that there exists a subset $K=\left\{k_{j}: j \in\right.$ $\mathbb{N}\}$ of $\mathbb{N}$ with $\delta(K)=1$ such that $x_{k_{j}} \stackrel{\text { ru }}{\rightarrow} x(e)$. Then there exists a sequence $\left(\epsilon_{j}\right)_{j \in \mathbb{N}}$ of positive reals with $\epsilon_{j} \downarrow 0$ such that $\left|x_{k_{j}}-x\right| \leq \epsilon_{j} e$ for all $j \in \mathbb{N}$.

We set

$$
y_{n}= \begin{cases}x_{n}, & n \in K \\ x, & n \notin K\end{cases}
$$

and $\eta_{n}=\epsilon_{j}$ for $k_{j-1}<n \leq k_{j}(j=1,2, \ldots)$, where $k_{0}=0$.

Then, $x_{n}=y_{n}$ a.a.n., $\eta_{n} \downarrow 0$, and $\left|y_{n}-x\right| \leq \eta_{n} e$ for all $n \in \mathbb{N}$. Hence $y_{n} \stackrel{\text { ru }}{\longrightarrow} x(e)$.

(3) $\Rightarrow(1)$ is trivial.

Corollary 8. Let $E$ be an Archimedean Riesz space. Then every monotone statistically relatively uniformly convergent sequence in $E$ is relatively uniformly convergent.

Proof. Suppose that $\left(x_{n}\right)_{n \in \mathbb{N}}$ is an increasing sequence in $E$ and $x_{n} \stackrel{\text { st }}{\rightarrow} x(e)$ for some $x \in X$ and $e \in E_{+}$. It follows from [16, Corollary 5] that $\sup _{n \in \mathbb{N}} x_{n}$ exists and equals $x$. By Theorem 7 , there exists a subset $K=\left\{k_{j}: j \in \mathbb{N}\right\}$ of $\mathbb{N}$ with $\delta(K)=1$ such that $x_{k_{j}} \stackrel{\text { ru }}{\rightarrow} x(e)$. Thus, we get a sequence $\left(\epsilon_{j}\right)_{j \in \mathbb{N}}$ of positive reals with $\epsilon_{j} \downarrow 0$ such that $\left|x_{k_{j}}-x\right| \leq \epsilon_{j} e$ for all $j \in \mathbb{N}$. We set

$$
\eta_{n}= \begin{cases}\epsilon_{1}, & 1 \leq n \leq k_{1} \\ \epsilon_{j-1}, & k_{j-1}<n \leq k_{j}(j=2,3, \ldots) .\end{cases}
$$


Then $\eta_{n} \downarrow 0$. We set $u=\left(x-x_{1}\right) \vee e$. Then, for $1 \leq n \leq k_{1}$, we have

$$
x-x_{n} \leq x-x_{1} \leq \eta_{1} u
$$

For $k_{j-1}<n \leq k_{j}(j=2,3, \ldots)$, we have

$$
x-x_{n} \leq x-x_{k_{j-1}} \leq \eta_{n} e \leq \eta_{n} u
$$

Hence, $x_{n} \stackrel{\text { ru }}{\longrightarrow} x(u)$. This completes the proof.

Let $E$ be a Riesz space and let $e \in E_{+}$. Following [13], recall that a sequence $\left(x_{n}\right)_{n \in \mathbb{N}}$ in $E$ is called an e-uniform Cauchy sequence whenever, for every $\epsilon>0$, there exists a natural number $N$ such that $\left|x_{m}-x_{n}\right| \leq \epsilon e$ holds for all $m, n \geq N$. We say that a sequence $\left(x_{n}\right)_{n \in \mathbb{N}}$ is uniform Cauchy if it is $e$ uniform Cauchy for some $e \in E_{+}$.

Definition 9. Let $E$ be a Riesz space and let $e \in E_{+}$. We say that a sequence $\left(x_{n}\right)_{n \in \mathbb{N}}$ in $E$ is a statistically e-uniform Cauchy sequence whenever, for every $\epsilon>0$, there exists a natural number $N$ such that $\left|x_{n}-x_{N}\right| \leq \epsilon e$, a.a.n. We say that a sequence $\left(x_{n}\right)_{n \in \mathbb{N}}$ is statistically uniform Cauchy if it is statistically $e$-uniform Cauchy for some $e \in E_{+}$.

Following [15], a sequence $\left(x_{n}\right)_{n \in \mathbb{N}}$ in a Riesz space $E$ is said to be statistically order bounded if there exist $y, z \in E$ such that $y \leq x_{n} \leq z$, a.a.n. It is easy to see that every statistically $e$-uniform Cauchy sequence is statistically order bounded for all $e \in E_{+}$. It should be noted that a statistically uniform Cauchy sequence need not be uniform Cauchy as can be seen in the following example.

Example 10. Let $K=\left\{k^{2}: k \in \mathbb{N}\right\}$. For each $n \in \mathbb{N}$, define $f_{n} \in C[0,1]$ by

$$
f_{n}= \begin{cases}n, & n \in K \\ \frac{e}{n}, & n \notin K,\end{cases}
$$

where $e(t)=t, t \in[0,1]$. Then $f_{n}=e / n$, a.a.n. and hence $f_{n} \stackrel{\text { st }}{\rightarrow} 0(e)$. Thus, we see that the sequence $\left(f_{n}\right)_{n \in \mathbb{N}}$ is statistically $e$-uniform Cauchy, but not uniform Cauchy since $\sup _{n \in \mathbb{N}}\left\|f_{n}\right\|=\infty$.

Theorem 11. Let $E$ be a Riesz space and $\in \in E_{+}$. The following are equivalent for a sequence $\left(x_{n}\right)_{n \in \mathbb{N}}$ in $E$ :

(1) $\left(x_{n}\right)_{n \in \mathbb{N}}$ is a statistically e-uniform Cauchy sequence.

(2) There exists a subset $K=\left\{k_{j}: j \in \mathbb{N}\right\}$ of $\mathbb{N}$ with $\delta(K)=$ 1 such that $\left(x_{k_{j}}\right)_{j \in \mathbb{N}}$ is an e-uniform Cauchy sequence.

Proof.

$(1) \Rightarrow(2)$. For each $k \in \mathbb{N}$, by (1), we get a natural number $N_{k}$ such that

$$
\delta\left(\left\{n \in \mathbb{N}:\left|x_{n}-x_{N_{k}}\right| \leq \frac{1}{k} e\right\}\right)=1
$$

Let $A_{k}=\left\{n \in \mathbb{N}:\left|x_{n}-x_{N_{k}}\right| \leq(1 / k) e\right\}(k=1,2, \ldots)$. It follows from Lemma 3 that there exists a subset $K \subseteq \mathbb{N}$ with $\delta(K)=1$ such that $\left|K \backslash A_{k}\right|<\infty$ for all $k \in \mathbb{N}$. Let $\epsilon>0$. Choose $k_{0}$ such that $1 / k_{0}<\epsilon / 2$. Since the set $K \backslash A_{k_{0}}$ is finite, there exists a natural number $N$ such that, for all $m, n>N, m, n \in K$, we have

$$
\begin{aligned}
& \left|x_{m}-x_{N_{k_{0}}}\right| \leq \frac{1}{k_{0}} e \leq \frac{\epsilon}{2} e, \\
& \left|x_{n}-x_{N_{k_{0}}}\right| \leq \frac{1}{k_{0}} e \leq \frac{\epsilon}{2} e .
\end{aligned}
$$

Hence, we get

$$
\left|x_{m}-x_{n}\right| \leq \epsilon e .
$$

This means that the sequence $\left(x_{n}\right)_{n \in \mathbb{N}}$ is an $e$-uniform Cauchy sequence along $K$.

$(2) \Rightarrow(1)$. Let $K$ be as stated in (2). Let $\epsilon>0$. There exists a natural number $j_{0}$ such that

$$
\left|x_{k_{i}}-x_{k_{j}}\right| \leq \epsilon e, \quad \forall i, j \geq j_{0} .
$$

This implies that

$$
\left\{k_{j}: j \geq j_{0}\right\} \subseteq\left\{n \in \mathbb{N}:\left|x_{n}-x_{k_{j_{0}}}\right| \leq \epsilon e\right\} .
$$

Hence, we get that $\left|x_{n}-x_{k_{j_{0}}}\right| \leq \epsilon e$, a.a.n. This completes the proof.

Corollary 12. Let $E$ be a Riesz space. Then every monotone statistically e-uniform Cauchy sequence is e-uniform Cauchy for all $e \in E_{+}$.

Proof. Suppose that $\left(x_{n}\right)_{n \in \mathbb{N}}$ is an increasing statistically $e$ uniform Cauchy sequence in $E$. It follows from Theorem 11 that there exists a strictly increasing sequence $\left(k_{j}\right)_{j \in \mathbb{N}}$ of positive integers such that $\left(x_{k_{j}}\right)_{j \in \mathbb{N}}$ is an $e$-uniform Cauchy sequence. Let $\epsilon>0$. Then there exists a natural number $j_{0}$ such that $\left|x_{k_{i}}-x_{k_{j}}\right| \leq \epsilon e$ for all $i, j \geq j_{0}$. For all $m>n \geq k_{j_{0}}$, if $k_{j} \leq n<m<k_{j+1}$ for some $j \geq j_{0}$, then we have

$$
0 \leq x_{m}-x_{n} \leq x_{k_{j+1}}-x_{k_{j}} \leq \epsilon e .
$$

If $k_{j} \leq n<k_{j+1}$ for some $j \geq j_{0}$ and $k_{i} \leq m<k_{i+1}$ for some $i>j$, then we have

$$
0 \leq x_{m}-x_{n} \leq x_{k_{i+1}}-x_{k_{j}} \leq \epsilon e .
$$

Hence, $\left(x_{n}\right)_{n \in \mathbb{N}}$ is $e$-uniform Cauchy.

Let $E$ be a Riesz space and $0<e \in E$. Recall in [16] that a sequence $\left(x_{n}\right)_{n \in \mathbb{N}}$ in $E$ is said to be statistically e-uniform preCauchy if

$$
\lim _{n \rightarrow \infty} \frac{1}{n^{2}}\left|\left\{(i, j): i, j \leq n,\left(\left|x_{i}-x_{j}\right|-\epsilon e\right)^{+}>0\right\}\right|=0
$$

for every $\epsilon>0$. We say that a sequence $\left(x_{n}\right)_{n \in \mathbb{N}}$ is statistically uniform pre-Cauchy if it is statistically $e$-uniform pre-Cauchy for some $0<e \in E$. 
Corollary 13. Every statistically e-uniform Cauchy sequence in a Riesz space $E$ is statistically e-uniform pre-Cauchy for all $0<e \in E$.

Proof. Let $\left(x_{n}\right)_{n \in \mathbb{N}}$ be a statistically $e$-uniform Cauchy sequence in $E$. By Theorem 11, there exists a subset $K \subseteq \mathbb{N}$ with $\delta(K)=1$ such that $\left(x_{n}\right)_{n \in K}$ is $e$-uniform Cauchy along $K$. Let $\epsilon>0$. There exists a subset $A \subseteq K$ such that $K \backslash A$ is finite and

$$
A \times A \subseteq\left\{(j, k):\left|x_{j}-x_{k}\right| \leq \epsilon e\right\} .
$$

Note that, for each $n \in \mathbb{N}$, we have

$$
\left|A_{n}\right|^{2}=|\{(j, k) \in A \times A: j, k \leq n\}| .
$$

Hence,

$$
\frac{1}{n^{2}}\left|A_{n}\right|^{2} \leq \frac{1}{n^{2}}\left|\left\{(j, k): j, k \leq n,\left|x_{j}-x_{k}\right| \leq \epsilon e\right\}\right| .
$$

Since $\delta(A)=1$, we get, by letting $n \rightarrow \infty$,

$$
\lim _{n \rightarrow \infty} \frac{1}{n^{2}}\left|\left\{(j, k): j, k \leq n,\left|x_{j}-x_{k}\right| \leq \epsilon e\right\}\right|=1 .
$$

We are done.

The following example shows that a statistically uniform pre-Cauchy sequence need not be statistically uniform Cauchy.

Example 14. We take [19, Example 8].

Let

$$
x_{k}=\sum_{i=1}^{m} \frac{1}{i}, \quad m ! \leq k<(m+1) !, k, m=1,2, \ldots .
$$

Then $\left(x_{k}\right)_{k \in \mathbb{N}}$ is increasing and tends to $\infty$. Hence, the sequence $\left(x_{k}\right)_{k \in \mathbb{N}}$ has no convergent subsequences and so is not statistically convergent. It follows from [17, Theorem 1] that $\left(x_{k}\right)_{k \in \mathbb{N}}$ is not statistically Cauchy and hence is not statistically uniform Cauchy. It is proved in [19, Example 8] that $\left(x_{k}\right)_{k \in \mathbb{N}}$ is statistically pre-Cauchy. This means that $\left(x_{k}\right)_{k \in \mathbb{N}}$ is statistically 1-uniform pre-Cauchy.

Theorem 15. Let $E$ be an Archimedean Riesz space ande $\in E_{+}$. Then

(1) every statistically e-uniformly convergent sequence in $E$ is statistically e-uniform Cauchy;

(2) if, moreover, $E$ is Dedekind $\sigma$-complete, then every monotone statistically e-uniform Cauchy sequence in $E$ is statistically e-uniformly convergent.

Proof. (1) Suppose that $x_{n} \stackrel{\text { st }}{\rightarrow} x(e)$. Let $\epsilon>0$. Then $\delta(\{n \in \mathbb{N}$ : $\left.\left.\left|x_{n}-x\right| \leq(\epsilon / 2) e\right\}\right)=1$. Let $K=\left\{n \in \mathbb{N}:\left|x_{n}-x\right| \leq(\epsilon / 2) e\right\}$ and $N=\min _{n \in K} n$. Then, for every $n \in K$, we have

$$
\left|x_{n}-x_{N}\right| \leq\left|x_{n}-x\right|+\left|x_{N}-x\right| \leq \frac{\epsilon}{2} e+\frac{\epsilon}{2} e=\epsilon e .
$$

This implies that $K \subseteq\left\{n \in \mathbb{N}:\left|x_{n}-x_{N}\right| \leq \epsilon e\right\}$ and hence $\delta\left(\left\{n \in \mathbb{N}:\left|x_{n}-x_{N}\right| \leq \epsilon e\right\}\right)=1$.
(2) Let $\left(x_{n}\right)_{n \in \mathbb{N}}$ be an increasing, statistically $e$-uniform Cauchy sequence in $E$. Then $\left(x_{n}\right)_{n \in \mathbb{N}}$ is statistically order bounded. Since $\left(x_{n}\right)_{n \in \mathbb{N}}$ is increasing, we see that $\left(x_{n}\right)_{n \in \mathbb{N}}$ is order bounded. Since $E$ is Dedekind $\sigma$-complete, then $\sup _{n \in \mathbb{N}} x_{n}$ exists and is denoted by $x$.

Let $\epsilon>0$. Then there is a natural number $N$ such that $\left|x_{n}-x_{N}\right| \leq \epsilon e$, a.a.n. Let $K=\left\{n \in \mathbb{N}:\left|x_{n}-x_{N}\right| \leq \epsilon e\right\}$ and $A=\{n \in K: n \geq N\}$. Then $\delta(K)=\delta(A)=1$. Let us fix any $n \in A$. Then, for any $m \geq n, m \in A$, we have

$$
x_{m}-x_{n} \leq x_{m}-x_{N} \leq \epsilon e .
$$

That is,

$$
x_{m} \leq x_{n}+\epsilon e, \quad \forall m \in\{k \in A: k \geq n\} .
$$

Since $\left(x_{n}\right)_{n \in \mathbb{N}}$ is increasing, we get $x=\sup _{m \in\{k \in A: k \geq n\}} x_{m}$. Hence, we have

$$
x \leq x_{n}+\epsilon e, \quad \forall n \in A .
$$

This means

$$
0 \leq x-x_{n} \leq \epsilon e, \text { a.a.n. }
$$

Thus,

$$
x_{n} \stackrel{\text { st }}{\rightarrow} x(e)
$$

It follows from Theorem 15 and Corollary 13 that every statistically $e$-uniformly convergent sequence is statistically $e$ uniform pre-Cauchy for all $e \in E_{+}$. The next result suggests that, under certain circumstances, the converse is also true. This result is an analogue of [19, Theorem 7].

Theorem 16. Let $E$ be an Archimedean Riesz space and $\in E_{+}$. Assume that $\left(x_{n}\right)_{n \in \mathbb{N}}$ is a statistically e-uniform pre-Cauchy sequence in $E$. If $\left(x_{n}\right)_{n \in \mathbb{N}}$ has a subsequence $\left(x_{n}\right)_{n \in K}$ which converges e-uniformly to $x \in E$ and $\liminf _{n \rightarrow \infty}\left(\left|K_{n}\right| / n\right)>0$. Then $x_{n} \stackrel{s t}{\rightarrow} x(e)$.

Proof. Let $\epsilon>0$. Since $\left(x_{n}\right)_{n \in K}$ converges $e$-uniformly to $x$, there exists a subset $A \subseteq K$ such that $K \backslash A$ is finite and

$$
\left|x_{j}-x\right| \leq \frac{\epsilon}{2} e, \quad \forall j \in A .
$$

We set $B=\left\{k \in \mathbb{N}:\left(\left|x_{k}-x\right|-\epsilon e\right)^{+} \neq 0\right\}$. Then, for $(j, k) \in$ $A \times B$, we have

$$
\left(\left|x_{j}-x_{k}\right|-\frac{\epsilon}{2} e\right)^{+} \neq 0 \text {. }
$$

That is,

$$
A \times B \subseteq\left\{(j, k):\left(\left|x_{j}-x_{k}\right|-\frac{\epsilon}{2} e\right)^{+} \neq 0\right\} .
$$

This implies

$$
\begin{aligned}
& \frac{\left|A_{n}\right|}{n} \frac{\left|B_{n}\right|}{n} \\
& \leq \frac{\left|\left\{(j, k):\left(\left|x_{j}-x_{k}\right|-(\epsilon / 2) e\right)^{+} \neq 0, j, k \leq n\right\}\right|}{n^{2}} .
\end{aligned}
$$

Letting $n \rightarrow \infty$, we get $\delta(B)=0$. This completes the proof. 


\section{Relationship to Statistical Order Convergence and Norm Statistical Convergence}

Let us recall in [13] that a sequence $\left(x_{n}\right)_{n \in \mathbb{N}}$ in a Riesz space $E$ is order convergent to $x \in E$ whenever there exists a sequence $p_{n} \downarrow 0$ in $E$ such that $\left|x_{n}-x\right| \leq p_{n}$ holds for all $n$.

Definition 17 (see [15]). Let $\left(x_{n}\right)_{n \in \mathbb{N}}$ be a sequence in a Riesz space $E$. If there exists a set $K=\left\{k_{j}\right\}_{j \in \mathbb{N}}$ with $\delta(K)=1$ such that $\left(x_{k_{j}}\right)_{j \in \mathbb{N}}$ is increasing and $\sup _{j \in \mathbb{N}} x_{k_{j}}=x$ for some $x \in$ $E$, then one writes $x_{n} \uparrow^{\text {st }} x$. Similarly, if $\left(x_{k_{j}}\right)_{j \in \mathbb{N}}$ is decreasing and inf ${ }_{j \in \mathbb{N}} x_{k_{j}}=x$ for some $x \in E$, then one writes $x_{n} \downarrow^{\text {st }} x$. If $x_{n} \uparrow^{\text {st }} x$ or $x_{n} \downarrow^{\text {st }} x$, then one said that $\left(x_{n}\right)_{n \in \mathbb{N}}$ is statistically monotone convergent to $x$.

Sençimen and Pehlivan [15] modified the definition of the usual order convergence and introduced the notion of statistical order convergence as follows.

Definition 18 (see [15]). Let $E$ be a Riesz space. A sequence $\left(x_{n}\right)_{n \in \mathbb{N}}$ in $E$ is statistically order convergent to $x \in E$ provided that there exist a sequence $\left(p_{n}\right)_{n \in \mathbb{N}}$ such that $p_{n} \downarrow^{\text {st }} 0$ and a subset $K$ of $\mathbb{N}$ with $\delta(K)=1$ such that $\left|x_{n}-x\right| \leq p_{n}$ for every $n \in K$. One writes $x_{n} \stackrel{\text { st-ord }}{\longrightarrow} x$.

Remark 19. It is easy to see that $x_{n} \stackrel{\text { st-ord }}{\longrightarrow} x$ if and only if there exists a sequence $\left(p_{n}\right)_{n \in \mathbb{N}}$ in $E$ with $p_{n} \downarrow 0$ such that $\left|x_{n}-x\right| \leq p_{n}$, a.a.n.

Theorem 20. Let $\left(x_{n}\right)_{n \in \mathbb{N}}$ be a sequence in $E, x \in E$ and $e \in$ $E_{+}$. Then $x_{n} \stackrel{r u}{\longrightarrow} x(e)$ if and only if $x_{n} \stackrel{\text { st-ord }}{\longrightarrow} x$ and $\left(x_{n}\right)_{n \in \mathbb{N}}$ is an e-uniform Cauchy sequence.

Proof. The necessary part is trivial. We only prove the sufficient part.

Suppose that $x_{n} \stackrel{\text { st-ord }}{\longrightarrow} x$ and $\left(x_{n}\right)_{n \in \mathbb{N}}$ is $e$-uniform Cauchy. By Remark 19, there exists a sequence $\left(p_{n}\right)_{n \in \mathbb{N}}$ in $E$ with $p_{n} \downarrow 0$ such that $\left|x_{n}-x\right| \leq p_{n}$, a.a.n. Let $K=\{n \in \mathbb{N}$ : $\left.\left|x_{n}-x\right| \leq p_{n}\right\}$. Then $\delta(K)=1$. Let $\epsilon>0$. Then there exists a natural number $N$ such that $\left|x_{m}-x_{n}\right| \leq \epsilon e$ for all $m, n \geq N$. Let us fix any $n \geq N$. Then, for any $m \in\{j \in K: j \geq n\}$, we have

$$
\left|x_{n}-x\right| \leq\left|x_{n}-x_{m}\right|+\left|x_{m}-x\right| \leq \epsilon e+p_{m} .
$$

Note that $\inf _{m \in K, m \geq n} p_{m}=0$. Hence, we get $\left|x_{n}-x\right| \leq \epsilon e$ for all $n \geq N$. This finishes the proof.

Theorem 21. Let $\left(x_{n}\right)_{n \in \mathbb{N}}$ be a sequence in $E, x \in E$ and $\in E_{+}$. Then $x_{n} \stackrel{\text { st }}{\rightarrow} x(e)$ if and only if $x_{n} \stackrel{\text { st-ord }}{\longrightarrow} x$ and $\left(x_{n}\right)_{n \in \mathbb{N}}$ is a statistically e-uniform Cauchy sequence.

Proof. It suffices to prove the sufficient part. Assume that $x_{n} \stackrel{\text { st-ord }}{\longrightarrow} x$ and $\left(x_{n}\right)_{n \in \mathbb{N}}$ is a statistically $e$-uniform Cauchy sequence. By [15, Theorem 5] and Theorem 7, we obtain a subset $K=\left(k_{j}\right)_{j \in \mathbb{N}}$ of $\mathbb{N}$ with $\delta(K)=1$ such that the sequence $\left(x_{k_{j}}\right)_{j \in \mathbb{N}}$ converges in order to $x$ and is $e$-uniform Cauchy. Let $\epsilon>0$. There exists a natural number $j_{0}$ such that

$$
\left|x_{k_{i}}-x_{k_{j}}\right| \leq \epsilon e, \quad \forall i, j \geq j_{0} \text {. }
$$

Let us fix any $j \geq j_{0}$. Letting $i \rightarrow \infty$, we get

$$
\left|x-x_{k_{j}}\right| \leq \epsilon e .
$$

This means that $x_{k_{j}} \stackrel{\text { ru }}{\rightarrow} x(e)$. It follows from Theorem 7 that $x_{n} \stackrel{\text { st }}{\rightarrow} x(e)$.

Theorem 22. Let $E$ be an Archimedean Riesz space. The following statements are equivalent:

(1) Statistical order convergence is stable.

(2) Statistical order convergence and statistically relatively uniform convergence are equivalent.

Proof.

$(1) \Rightarrow(2)$. Suppose that $x_{n} \stackrel{\text { st-ord }}{\longrightarrow} 0$. Then, by (1), there exists a sequence $\left(\lambda_{n}\right)_{n \in \mathbb{N}}$ of reals with $0 \leq \lambda_{n} \uparrow \infty$ such that $\lambda_{n} x_{n} \stackrel{\text { st-ord }}{\longrightarrow} 0$. By Remark 19, there exists a sequence $\left(p_{n}\right)_{n \in \mathbb{N}}$ with $p_{n} \downarrow 0$ such that

$$
\lambda_{n}\left|x_{n}\right| \leq p_{n}, \quad \text { a.a.n. }
$$

This means

$$
\left|x_{n}\right| \leq \frac{1}{\lambda_{n}} p_{1}, \quad \text { a.a.n. }
$$

Hence, $x_{n} \stackrel{\text { st }}{\rightarrow} 0\left(p_{1}\right)$ and then $x_{n} \stackrel{\text { sru }}{\rightarrow} 0$.

$(2) \Rightarrow(1)$. Assume that $x_{n} \stackrel{\text { st-ord }}{\longrightarrow} 0$. By (2), we see that $x_{n} \stackrel{\text { sru }}{\longrightarrow}$ 0 . Then, there exist $e \in E_{+}$and a sequence $\epsilon_{n} \downarrow 0$ such that

$$
\left|x_{n}\right| \leq \epsilon_{n} e, \text { a.a.n. }
$$

Thus, we get $\left(1 / \sqrt{\epsilon_{n}}\right) x_{n} \stackrel{\text { st-ord }}{\longrightarrow} 0$. And we are done.

Note that statistical order convergence and statistically relatively uniform convergence are not equivalent in general, as can be seen in the following example.

Example 23. Let us take a counterexample in [13, Exercise 16.18 , p. 90]. For each $n \in \mathbb{N}$, we define $f_{n} \in C[0,1]$ by $f_{n}(t)=$ 1 on $[0,1 / n]$ and $f_{n}(t)=0$ on $[2 / n, 1]$ and $f_{n}(t)$ is linear on $[1 / n, 2 / n]$. Clearly, $f_{n} \downarrow 0$. Moreover, there is no sequence $0 \leq$ $\lambda_{n} \uparrow \infty$ such that $\lambda_{n} f_{n} \stackrel{\text { st-ord }}{\longrightarrow} 0$. Otherwise, it follows from [15, Theorem 5] that there exists a subset $K=\left\{k_{j}: j \in \mathbb{N}\right\}$ of $\mathbb{N}$ with $\delta(K)=1$ such that the sequence $\left(\lambda_{k_{j}} f_{k_{j}}\right)_{j \in \mathbb{N}}$ is order convergent 0 . Hence, there exists a sequence $\left(p_{j}\right)_{j \in \mathbb{N}}$ in $E$ with $p_{j} \downarrow 0$ so that $\lambda_{k_{j}} f_{k_{j}} \leq p_{j}$ for all $j \in \mathbb{N}$. This implies

$$
\begin{aligned}
1=f_{k_{j}}(0) \leq \frac{1}{\lambda_{k_{j}}} p_{j}(0) \leq \frac{1}{\lambda_{k_{j}}} p_{1}(0) \longrightarrow & 0 \\
& (j \longrightarrow \infty) .
\end{aligned}
$$

This contradiction suggests that statistical order convergence in $C[0,1]$ is not stable. Then Theorem 22 gives the conclusion. 
Following [12], a sequence $\left(x_{n}\right)_{n \in \mathbb{N}}$ in a Banach space $E$ is said to be norm statistically convergent to $x \in E$ provided that $\delta\left(\left\{n \in \mathbb{N}:\left\|x_{n}-x\right\| \geq \epsilon\right\}\right)=0$ for all $\epsilon>0$. It is clear that a sequence $\left(x_{n}\right)_{n \in \mathbb{N}}$ in a Banach lattice $E$ is norm statistically convergent whenever it is statistically relatively uniformly convergent. The sequence $\left(x_{n}\right)_{n \in \mathbb{N}}$ is said to be weakly statistically convergent to $x$ provided that the sequence $\left(\left\langle x^{*}, x_{n}-x\right\rangle\right)_{n \in \mathbb{N}}$ converges statistically to 0 for each $x^{*} \in X^{*}$. It is noted in [12] that if a sequence $\left(x_{n}\right)_{n \in \mathbb{N}}$ in a Banach space $E$ is norm statistically convergent to $x \in E$, then there exists a subset $K=\left(k_{j}\right)_{j \in \mathbb{N}} \subseteq \mathbb{N}$ with $\delta(K)=1$ such that $\left(x_{k_{j}}\right)_{j \in \mathbb{N}}$ converges in norm to $x$. Combining this observation, Theorems 11 and 7 with [14, Theorem 100.4], we obtain the following result.

Theorem 24. Let $\left(x_{n}\right)_{n \in \mathbb{N}}$ be a sequence in a Banach lattice $E$, $x \in E$ and $e \in E_{+}$. Then $x_{n} \stackrel{s t}{\rightarrow} x(e)$ if and only if $\left(x_{n}\right)_{n \in \mathbb{N}}$ is norm statistically convergent to $x$ and $\left(x_{n}\right)_{n \in \mathbb{N}}$ is a statistically e-uniform Cauchy sequence.

It is known [18] that, in $C(K)$-spaces, the relatively uniform convergence coincides with the norm convergence. It follows from Theorem 7 that, in $C(K)$-spaces, the statistically relatively uniform convergence coincides with the norm statistical convergence. In Example 23, utilizing Theorem 22, we show that statistical order convergence and statistically relatively uniform convergence are not equivalent in general. Now we construct a statistical order convergent sequence that is not statistically relatively uniformly convergent as follows.

Example 25. Let $K=\left\{k^{2}: k \in \mathbb{N}\right\}$. For each $n \in \mathbb{N}$, we define $f_{n} \in C[0,1]$ by

$$
f_{n}= \begin{cases}1, & n \in K \\ g_{n}, & n \notin K,\end{cases}
$$

where $g_{n}(t)=t^{n}, t \in[0,1], n=1,2, \ldots$

Obviously, $g_{n} \downarrow 0$ and hence $f_{n} \stackrel{\text { st-ord }}{\longrightarrow} 0$. Since $\left\|g_{n}\right\|=1$ for all $n \in \mathbb{N}$, we see that the sequence $\left(f_{n}\right)_{n \in \mathbb{N}}$ is not norm statistically convergent and hence not statistically relatively uniformly convergent.

Example 5 in [15] suggests that the statistical order convergence need not be norm statistically convergent. The following example suggests that the norm statistical convergence need not be statistically order convergent and hence not be statistically relatively uniformly convergent.

Example 26. We take the sequence $\left(f_{n}\right)_{n \in \mathbb{N}}$ in [14, Exercise 100.13] as follows.

Let $E$ be the normed Riesz space of all real continuous functions on [0.1] with $\|f\|=\int_{0}^{1}|f| d t$. We set

$$
\begin{aligned}
f_{1}\left(\frac{1}{2}\right) & =1, \\
\left\|f_{1}\right\| & =\frac{1}{2},
\end{aligned}
$$

$$
0 \leq f_{1} \leq 1
$$

$$
\begin{aligned}
f_{2}\left(\frac{1}{3}\right) & =f_{2}\left(\frac{2}{3}\right)=1, \\
\left\|f_{2}\right\| & =\frac{1}{2^{2}},
\end{aligned}
$$

$$
0 \leq f_{2} \leq 1
$$

and generally

$$
\begin{aligned}
f_{n}\left(\frac{1}{n+1}\right) & =f_{n}\left(\frac{2}{n+1}\right)=\cdots=f_{n}\left(\frac{n}{n+1}\right)=1, \\
\left\|f_{n}\right\| & =\frac{1}{2^{n}}, \\
0 & \leq f_{n} \leq 1,
\end{aligned}
$$

for $n=1,2, \ldots$

It is proved in [14, Exercise 100.13] that the sequence $\left(f_{n}\right)_{n \in \mathbb{N}}$ converges to 0 in norm and no subsequence of it converges to 0 in order.

We set $K=\left\{k^{2}: k \in \mathbb{N}\right\}$ and let

$$
g_{n}= \begin{cases}1, & n \in K \\ f_{n}, & n \notin K .\end{cases}
$$

Clearly, $\left(g_{n}\right)_{n \in \mathbb{N}}$ is norm statistically convergent to 0 . But $\left(g_{n}\right)_{n \in \mathbb{N}}$ is not statistically order convergent. Indeed, if $\left(g_{n}\right)_{n \in \mathbb{N}}$ is statistically order convergent to 0 , it follows from [15, Theorem 5] that there exists a subset $A$ of $\mathbb{N}$ with $\delta(A)=1$ such that $\left(g_{n}\right)_{n \in A}$ is order convergent to 0 . Hence, the subsequence $\left(f_{n}\right)_{n \in A \cap(\mathbb{N} \backslash K)}=\left(g_{n}\right)_{n \in A \cap(\mathbb{N} \backslash K)}$ is order convergent to 0 . This is a contradiction.

Example 27. We take the sequence $\left(x^{(r)}\right)_{r \in \mathbb{N}}$ in [20, Example 5] as follows.

Let

$$
\begin{aligned}
E & =\left\{\left(x_{n}\right)_{n \in \mathbb{N}} \in\left(\sum_{n} \oplus l_{\infty}\right)_{\infty}: x_{n}\right. \\
& \left.\in \mathcal{c}_{0} \text { except for finitely many } n\right\} .
\end{aligned}
$$

Then $E$ is ideal in $\left(\sum_{n} \oplus l_{\infty}\right)_{\infty}$. Define $x^{(r)}=\left(x_{n}^{(r)}\right)_{n \in \mathbb{N}} \in E$ for $r=1,2, \ldots$ by

$$
\begin{aligned}
x_{n}^{(r)}(k) & =\frac{1}{r} \quad(k=1,2, \ldots), \text { for } n \leq r, \\
x_{n}^{(r)} & =0, \quad \text { for } n>r .
\end{aligned}
$$

Then $\left\|x^{(r)}\right\|=1 / r \rightarrow 0(r \rightarrow \infty)$, but no subsequence of $\left(x^{(r)}\right)_{r \in \mathbb{N}}$ is relatively uniformly convergent.

Then, as in Example 26, we set $K=\left\{k^{2}: k \in \mathbb{N}\right\}$ and let

$$
y^{(r)}= \begin{cases}0, & r \in K \\ x^{(r)}, & r \notin K .\end{cases}
$$


Clearly, $\left(y^{(r)}\right)_{r \in \mathbb{N}}$ is norm statistically convergent to 0 . An argument similar to Example 26 shows that $\left(y^{(r)}\right)_{r \in \mathbb{N}}$ is not relatively uniformly convergent.

The following example illustrates that even a combination of statistical order convergence and norm statistical convergence does not imply the statistically relatively uniform convergence.

Example 28. Let us take Example 2 in [20].

Pick $g \in L_{1}[0,1]$ such that $g$ is continuous on $(0,1], g(t)>0$ for all $t \in(0,1]$, and $\lim _{t \rightarrow 0^{+}} g(t)=\infty$. Let $E$ be the Riesz subspace of $L_{1}[0,1]$ consisting of all $f$ such that, for some $\delta(f)>0$ and $\gamma(f) \in \mathbb{R}$, we have $f(t)=\gamma(f) g(t)$ for all $0 \leq t<\delta(f)$. Define a Riesz norm $\|\cdot\|$ on $E$ by setting

$$
\|f\|=\|f\|_{L_{1}}+|\gamma(f)| .
$$

Moore Jr. exhibited a sequence $\left(f_{n}\right)_{n \in \mathbb{N}}$ in $E$ such that $f_{n} \downarrow 0$ and $\lim _{n \rightarrow \infty}\left\|f_{n}\right\|=0$, but $f_{n} \nrightarrow 0(\mathrm{ru})$, as follows.

Let $f_{0}=g$. Define $f_{n}$ by induction so that

(a) $f_{n}$ is continuous on $(0,1]$,

(b) $0 \leq f_{n} \leq f_{n-1}$,

(c) $f_{n}(1 / k)=(1 / k) f_{0}(1 / k), k=1,2, \ldots, n$,

(d) $f_{n}(t)=(1 /(n+1)) g(t), t \in(0,1 /(n+1)]$,

(e) $\left\|f_{n}\right\|_{L_{1}} \leq(1 /(n+1))\|g\|_{L_{1}}$.

This implies that no subsequence of $\left(f_{n}\right)_{n \in \mathbb{N}}$ converges relatively uniformly to 0 .

Similarly, we set $K=\left\{k^{2}: k \in \mathbb{N}\right\}$ and let

$$
g_{n}= \begin{cases}0, & n \in K \\ f_{n}, & n \notin K .\end{cases}
$$

Clearly, $\left(g_{n}\right)_{n \in \mathbb{N}}$ is norm statistically convergent to 0 and $g_{n} \stackrel{\text { st-ord }}{\longrightarrow} 0$. But Theorem 7 yields that $\left(g_{n}\right)_{n \in \mathbb{N}}$ is not statistically relatively uniformly convergent to 0 . This is because no subsequence of $\left(f_{n}\right)_{n \in \mathbb{N}}$ converges relatively uniformly to 0 .

Theorem 29. Let $\left(x_{n}\right)_{n \in \mathbb{N}}$ be a monotone sequence in a Banach lattice E. Then

(1) $\left(x_{n}\right)_{n \in \mathbb{N}}$ is norm statistically convergent if and only if it is norm convergent;

(2) $\left(x_{n}\right)_{n \in \mathbb{N}}$ is weakly statistically convergent if and only if it is weakly convergent;

(3) $\left(x_{n}\right)_{n \in \mathbb{N}}$ is norm statistically convergent if and only if it is weakly statistically convergent.

In order to prove Theorem 29, we need two simple lemmas. The first lemma is an immediate consequence of [21, Proposition 1.1.6].

Lemma 30. If a sequence $\left(x_{n}\right)_{n \in \mathbb{N}}$ in a Banach lattice $E$ is increasing and converges norm statistically to $x \in E$, then $x=\sup _{n \in \mathbb{N}} x_{n}$.
Actually, Lemma 30 also holds for weakly statistical convergence as shown in the following.

Lemma 31. If a sequence $\left(x_{n}\right)_{n \in \mathbb{N}}$ in a Banach lattice $E$ is increasing and converges weakly statistically to $x \in E$, then $x=\sup _{n \in \mathbb{N}} x_{n}$.

Proof. Let $x^{*} \in\left(E^{*}\right)_{+}$. Then there exists a strictly increasing sequence $\left(k_{j}\right)_{j \in \mathbb{N}}$ of natural numbers so that $\lim _{j \rightarrow \infty}\left\langle x^{*}, x_{k_{j}}\right\rangle=\left\langle x^{*}, x\right\rangle$. For each $n \in \mathbb{N}$, we choose $k_{j_{0}}$ with $k_{j_{0}}>n$. Then, we have

$$
\left\langle x^{*}, x_{n}\right\rangle \leq\left\langle x^{*}, x_{k_{j}}\right\rangle, \quad \forall j>j_{0} .
$$

This means that

$$
\left\langle x^{*}, x_{n}\right\rangle \leq\left\langle x^{*}, x\right\rangle \text {. }
$$

Hence, $x$ is an upper bound of $\left(x_{n}\right)_{n \in \mathbb{N}}$.

Assume that $u$ is an arbitrary upper bound of $\left(x_{n}\right)_{n \in \mathbb{N}}$. Then, for every $x^{*} \in\left(E^{*}\right)_{+}$, we have

$$
\left\langle x^{*}, x_{n}\right\rangle \leq\left\langle x^{*}, u\right\rangle, \quad \forall n \in \mathbb{N} .
$$

Since the sequence $\left(\left\langle x^{*}, x_{n}\right\rangle\right)_{n \in \mathbb{N}}$ converges statistically to $\left(\left\langle x^{*}, x\right\rangle\right)_{n \in \mathbb{N}}$, we have

$$
\left\langle x^{*}, x\right\rangle \leq\left\langle x^{*}, u\right\rangle \text {. }
$$

This implies that $x \leq u$ and we are done.

Proof of Theorem 29. (1) Suppose that $\left(x_{n}\right)_{n \in \mathbb{N}}$ is increasing and norm statistically convergent to $x \in E$. By Lemma 30, we get $x=\sup _{n \in \mathbb{N}} x_{n}$. Let $\left(k_{j}\right)_{j \in \mathbb{N}}$ be a strictly increasing sequence of natural numbers so that $\lim _{j \rightarrow \infty} x_{k_{j}}=x$ in norm. Let $\epsilon>0$. Choose a natural number $j_{0}$ such that $\left\|x_{k_{j_{0}}}-x\right\|<\epsilon$. Then, for any $n>k_{j_{0}}$, we have

$$
0 \leq x-x_{n} \leq x-x_{k_{j_{0}}}
$$

This yields

$$
\left\|x-x_{n}\right\| \leq\left\|x-x_{k_{j_{0}}}\right\|<\epsilon .
$$

Hence, $\lim _{n \rightarrow \infty} x_{n}=x$ in norm

(2) Let $\left(x_{n}\right)_{n \in \mathbb{N}}$ be increasing and weakly statistically convergent to $x \in E$. By Lemma 31, we get $x=\sup _{n \in \mathbb{N}} x_{n}$. Let $x^{*} \in\left(E^{*}\right)_{+}$. Since $\left(x_{n}\right)_{n \in \mathbb{N}}$ converges weakly statistically to $x,\left(\left\langle x^{*}, x_{n}\right\rangle\right)_{n \in \mathbb{N}}$ converges statistically to $\left\langle x^{*}, x\right\rangle$. There exists a subset $K \subseteq \mathbb{N}$ with $\delta(K)=1$ such that $\lim _{n \in K}\left\langle x^{*}, x_{n}\right\rangle=$ $\left\langle x^{*}, x\right\rangle$. Let $\epsilon>0$. Choose $N \in K$ such that $\mid\left\langle x^{*}, x_{N}\right\rangle-$ $\left\langle x^{*}, x\right\rangle \mid<\epsilon$. Then, for all $n>N$, we have

$$
0 \leq x-x_{n} \leq x-x_{N}
$$

This implies that

$$
0 \leq\left\langle x^{*}, x-x_{n}\right\rangle \leq\left\langle x^{*}, x-x_{N}\right\rangle<\epsilon .
$$

Hence, $\left(x_{n}\right)_{n \in \mathbb{N}}$ converges weakly to $x$.

(3) follows from (1) and (2). 


\section{Conflicts of Interest}

The authors declare that there are no conflicts of interest regarding the publication of this paper.

\section{Acknowledgments}

This research is supported by the National Natural Science Foundation of China (Grant no. 11571069).

\section{References}

[1] A. Zygmund, Trigonometrical series, Chelsea Publishing Co., NY, USA, 2nd edition, 1952.

[2] H. Steinhaus, "Sur la convergence ordinaire et la convergence asymptotique," Colloquium Mathematicum, vol. 2, pp. 73-74, 1951.

[3] H. Fast, "Sur la convergence statistique," Colloquium Mathematicum, vol. 2, pp. 241-244, 1951.

[4] I. J. Schoenberg, "The integrability of certain functions and related summability methods," The American Mathematical Monthly, vol. 66, pp. 361-375, 1959.

[5] P. Erdös and G. Tenenbaum, "Sur les densitiés de certaines suites dentiers," Proceedings of the London Mathematical Society, vol. 59, no. 3, pp. 417-438, 1989.

[6] A. R. Freedman and J. J. Sember, "Densities and summability," Pacific Journal of Mathematics, vol. 95, no. 2, pp. 293-305, 1981.

[7] H. I. Miller, "A measure theoretical subsequence characterization of statistical convergence," Transactions of the American Mathematical Society, vol. 347, no. 5, pp. 1811-1819, 1995.

[8] J. A. Fridy and M. K. Khan, "Tauberian theorems via statistical convergence," Journal of Mathematical Analysis and Applications, vol. 228, no. 1, pp. 73-95, 1998.

[9] G. Di Maio and L. s. Kocinac, "Statistical convergence in topology," Topology and its Applications, vol. 156, no. 1, pp. 2845, 2008.

[10] I. J. Maddox, "Statistical convergence in a locally convex space," Mathematical Proceedings of the Cambridge Philosophical Society, vol. 104, no. 1, pp. 141-145, 1988.

[11] I. J. Maddox, "A Tauberian theorem for statistical convergence," Mathematical Proceedings of the Cambridge Philosophical Society, vol. 106, no. 2, pp. 277-280, 1989.

[12] J. Connor, M. Ganichev, and V. Kadets, "A characterization of Banach spaces with separable duals via weak statistical convergence," Journal of Mathematical Analysis and Applications, vol. 244, no. 1, pp. 251-261, 2000.

[13] W. A. J. Luxemburg and A. C. Zaanen, Riesz Spaces I, NorthHolland, Amsterdam-London, 1971.

[14] A. C. Zaanen, Riesz Spaces II, vol. 30, North-Holland, Amsterdam, 1983.

[15] C. Sençimen and S. Pehlivan, "Statistical order convergence in Riesz spaces," Mathematica Slovaca, vol. 62, no. 2, pp. 257-270, 2012.

[16] Z. Ercan, "A characterization of u-uniformly completeness of Riesz spaces in terms of statistical u-uniformly pre-completeness," Demonstratio Mathematica, vol. 42, no. 2, pp. 381-385, 2009.

[17] J. A. Fridy, "On statistical convergence," International Journal of Analysis and Applications, vol. 5, no. 4, pp. 301-313, 1985.
[18] H.-U. Schwarz, "Banach lattices and operators," Teubner-Texte zur Mathematik, 1984.

[19] J. Connor, J. Fridy, and J. Kline, "Statistically pre-Cauchy sequences," Analysis International Mathematical Journal of Analysis and its Applications, vol. 14, no. 4, pp. 311-317, 1994.

[20] L. C. Moore Jr., "A comparison of the relative uniform topology and the norm topology in a normed Riesz space," Pacific Journal of Mathematics, vol. 71, no. 1, pp. 107-118, 1977.

[21] P. Meyer-Nieberg, Banach Lattices, Springer, NY, USA, 1991. 


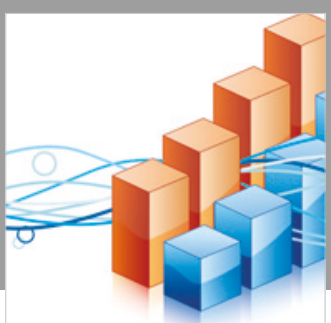

Advances in

Operations Research

\section{-n-m}
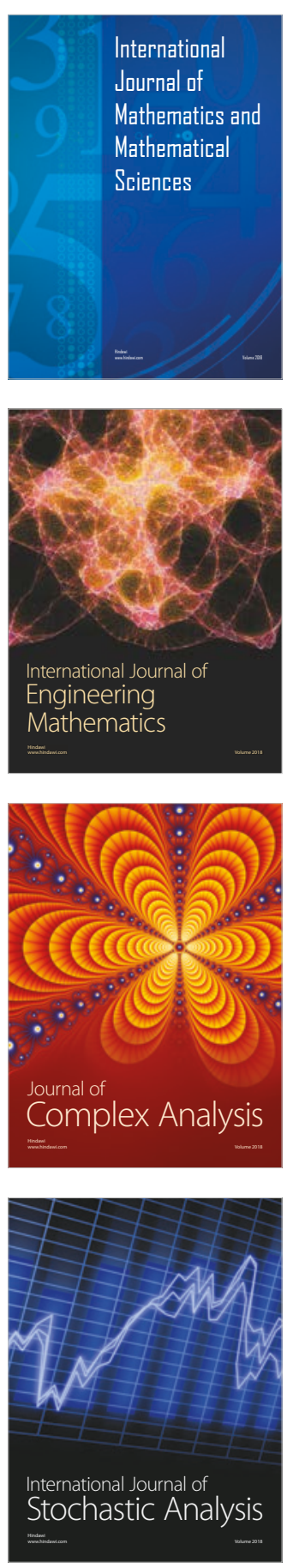
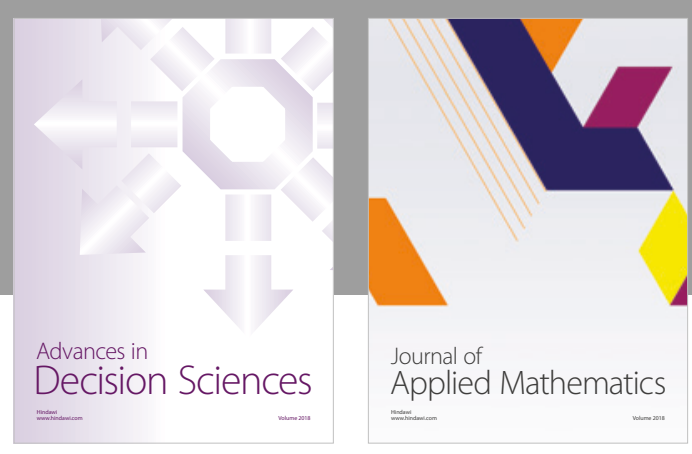

Journal of

Applied Mathematics
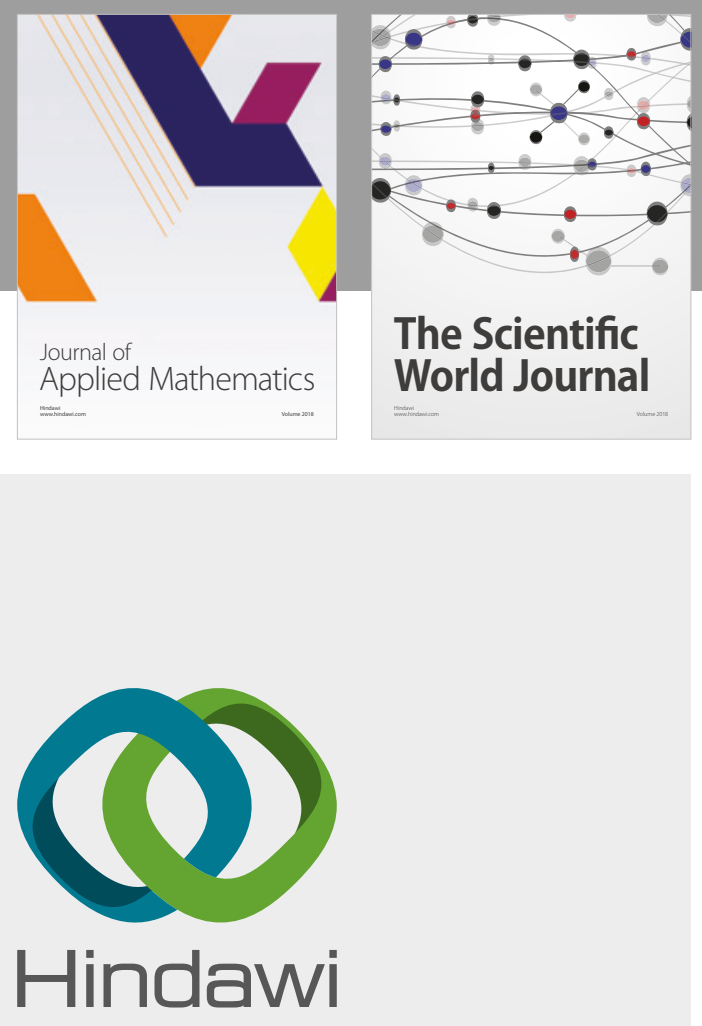

Submit your manuscripts at

www.hindawi.com

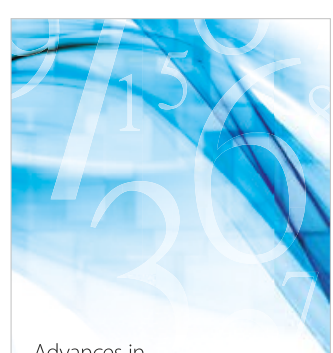

Advances in
Numerical Analysis
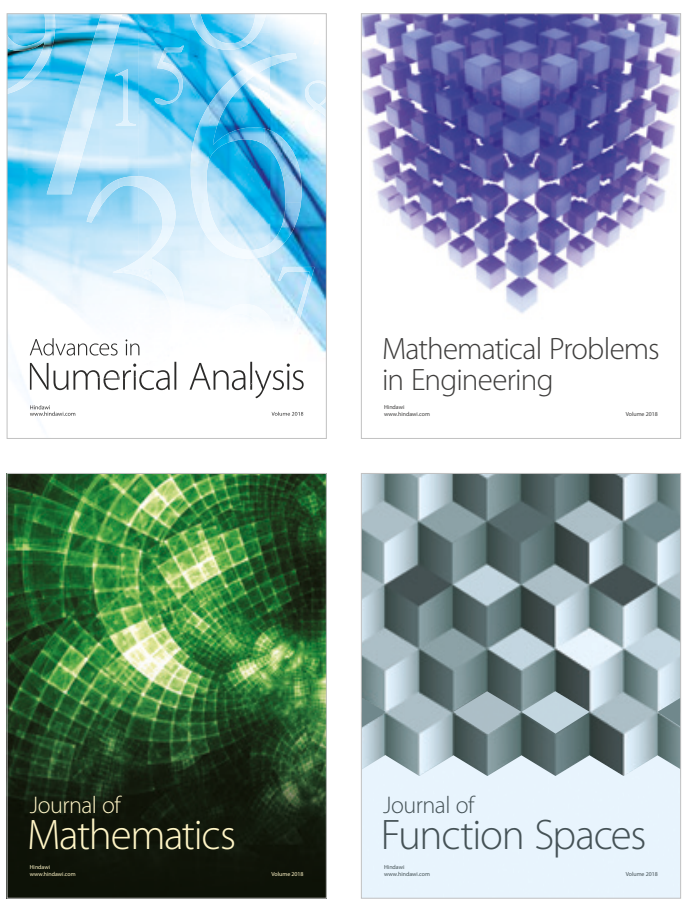

Mathematical Problems in Engineering

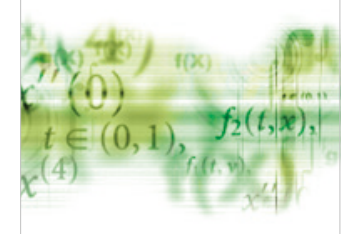

International Journal of

Differential Equations

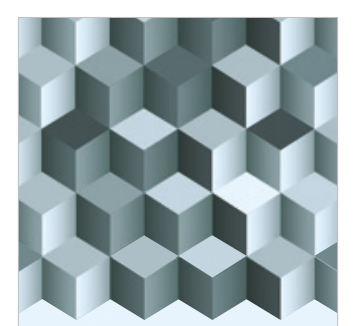

Journal of

Function Spaces

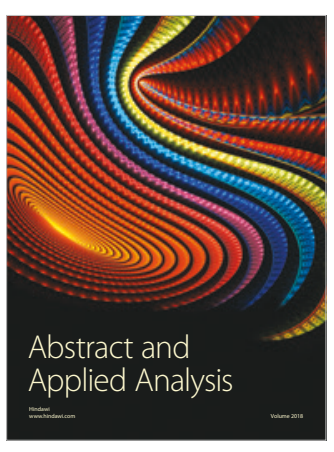

The Scientific

World Journal

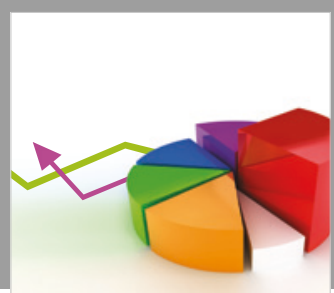

Journal of

Probability and Statistics
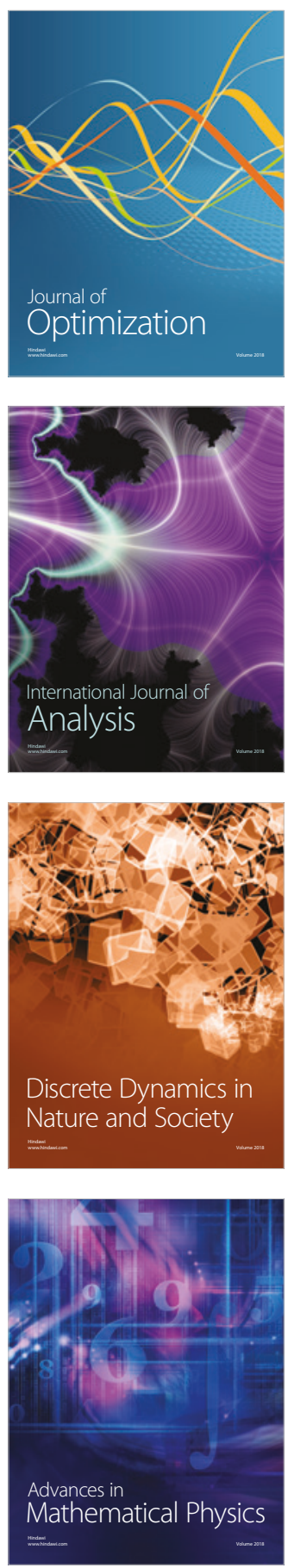